\title{
Reparación de catéteres tunelizados de hemodiálisis con moldes de silicona
}

\author{
Angélica Alarcia Martín, Mónica Brazález Tejerina, Cristina Franco Valdivieso, Laura Melero Salas, María \\ Jesús Rollán de la Sota
}

Enfermeras. Hospital Clínico Universitario de Valladolid

\section{Resumen}

Los pacientes que inician tratamiento renal sustitutivo son cada vez más añosos, lo que supone un mayor deterioro de la red vascular que influye negativamente en el desarrollo de la fístula arterio-venosa interna. Esto implica un aumento en el uso de catéteres tunelizados. El deterioro físico del catéter supone en ocasiones su recambio, comprometiendo la seguridad del paciente y aumentando innecesariamente el coste sanitario de su tratamiento. Para evitarlo se valora el uso del kit de reparación de catéteres peritoneales en los mencionados catéteres tunelizados, realizando un estudio retrospectivo en una población de tres pacientes a los que se detectó una fuga en el trayecto de su catéter. El tiempo de funcionamiento de estos catéteres tras su reparación fue variable, desde 9 meses hasta más de 3 años, estando uno de ellos en uso actualmente. Su retirada no estuvo relacionada en ningún caso con la técnica de reparación efectuada y sólo se registró una complicación menor en uno de los casos, por lo que se constata la eficacia de la técnica y el consiguiente ahorro en recursos materiales y humanos que supone.

PALABRAS CLAVE:

- REPARACIÓN

- CATÉTERES TUNELIZADOS

- HEMODIÁLISIS

- SILICONA

Correspondencia:

María Jesús Rollán de la Sota.

Servicio de Nefrología.

Hospital Clínico Universitario de Valladolid

C/Ramón y Cajal, s/n. 47005, Valladolid

E-mail:mjrollan@saludcastillyleon.es

\section{Repairing tunnelled haemodialysis catheters with silicon moulds}

\section{Abstract}

Patients starting renal replacement therapy are increasingly elderly, representing a further deterioration of the vascular network that is affecting the development of internal arteriovenous fistula. This implies an increase in the use of tunneled catheters. The physical deterioration of the catheter sometimes leads to its replacement, compromising patient safety and unnecessary increasing health costs of treatment. To avoid this, we evaluate the use of peritoneal catheter repair kits in tunneled catheters, performing a retrospective study in a population of three patients to whom a leak was detected in the path of their catheters. The running time of these catheters after repair was variable, ranging from 9 months to more than 3 years, being one of them still in use. Their withdrawal was not related in any way with the technique of repair performed and there was only a minor complication in one case, what confirms the effectiveness of the technique developed and the consequent saving in material and human resources.

\section{KEY-WORDS: \\ - REPARATION \\ - TUNNELED CATHETERS \\ - HEMODIALYSIS \\ - SILICONE}

\section{Introducción}

España ha experimentado un cambio demográfico en las últimas décadas que ha supuesto un envejecimiento de la población debido al aumento de la esperanza de vida, entre otros factores. Este envejecimiento supone el aumento de las enfermedades crónicas, incluyendo la 
enfermedad renal crónica (ERC). Por ello, los pacientes que inician tratamiento renal sustitutivo (TRS) con hemodiálisis (HD) son cada vez más añosos. Esto, unido a las alteraciones del árbol vascular que presentan, puede influir negativamente en el desarrollo de la fístula arterio-venosa interna (FAVI) ${ }^{1}$.

A pesar de estar establecidos como accesos vasculares de primera elección la FAVI y en segundo lugar la FAVI protésica ${ }^{2,3}$, el empleo de catéteres tanto temporales como tunelizados se ha incrementado enormemente en los últimos años. Esta situación ocasiona complicaciones, que repercuten y sobrecargan la labor de enfermería. Los mayores problemas que encontramos son derivados de su uso y estructura física.

El deterioro físico del catéter supone en ocasiones su recambio, lo que implica para el paciente un tratamiento altamente invasivo que afecta a su seguridad tanto a nivel físico como psicológico, además de unos ingresos hospitalarios y gastos innecesarios para el sistema sanitario, algunos de los cuales podrían ser subsanados con ciertas acciones llevadas a cabo en la sala de diálisis. Esto mantendría el catéter en buenas condiciones de uso y prolongaría su vida útil.

El objetivo del estudio será valorar la utilidad, eficacia y eficiencia de la técnica de reparación de catéteres tunelizados utilizada en nuestra unidad.

\section{Material y métodos}

Se realizó un estudio retrospectivo en una población de tres pacientes a los que se detectó una fuga en el trayecto externo del catéter. Dicho trayecto comprende desde el orificio de salida hasta la zona de bifurcación de las dos luces que lo componen. Se registró la fecha de reparación del catéter, los flujos efectivos en las sesiones posteriores a ésta, las complicaciones surgidas y la fecha y causas de retirada del catéter.

A continuación se detalla el procedimiento de actuación que se llevó a cabo:

1. Desconexión del paciente y finalización de la sesión de HD, taponando con una gasa la fuga, con las mayores condiciones asépticas posibles.

2. Realización de una analítica de control para valorar la situación dialítica del paciente.

3. Apertura del kit de reparación y de la silicona para el sellado, comercializado en un principio para la diálisis peritoneal (DP).
4. Colocación del molde rodeando el catéter e inyección de la silicona de forma inmediata.

de la solidificación de la silicona.

5. Inicio de la siguiente sesión de HD con flujos bajos que se fueron incrementando tras confirmar el correcto funcionamiento del catéter.

(Figuras: 1, 2, 3 y 4)

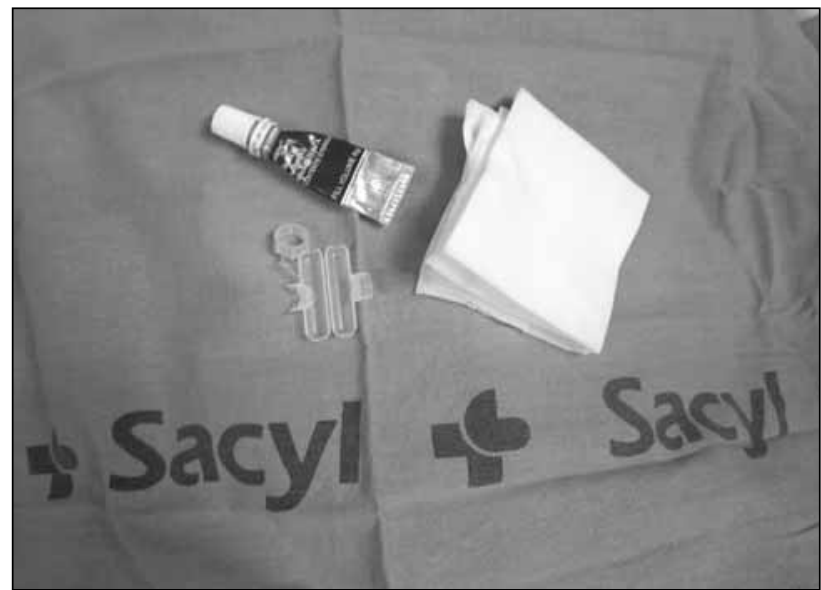

Figura 1.

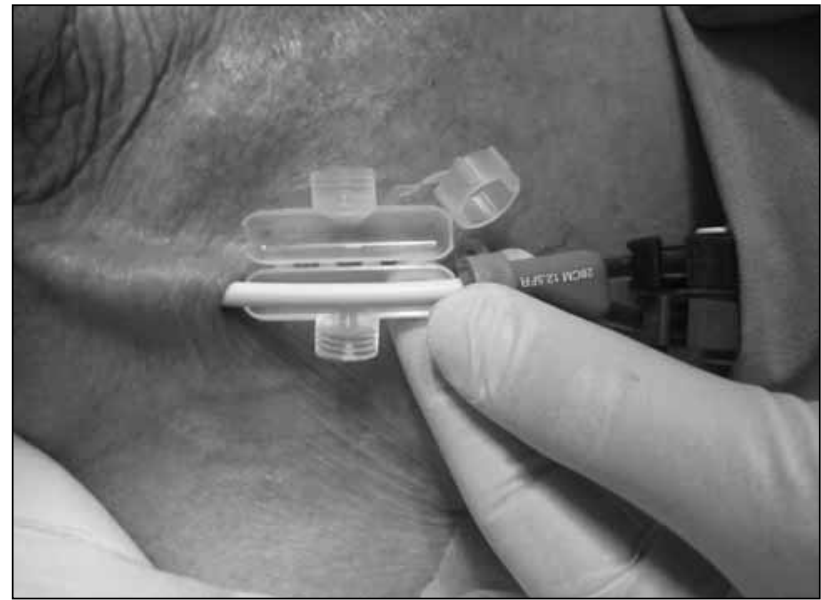

Figura 2.

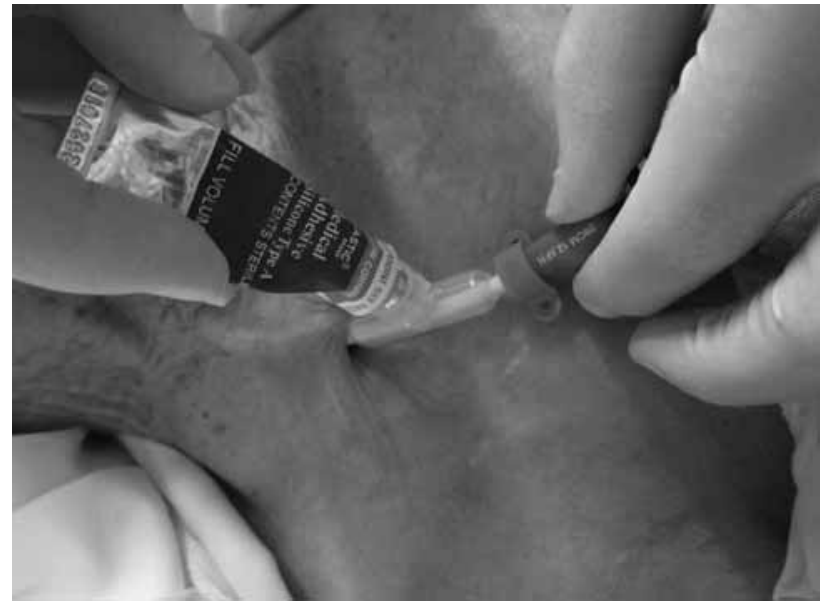

Figura 3. 


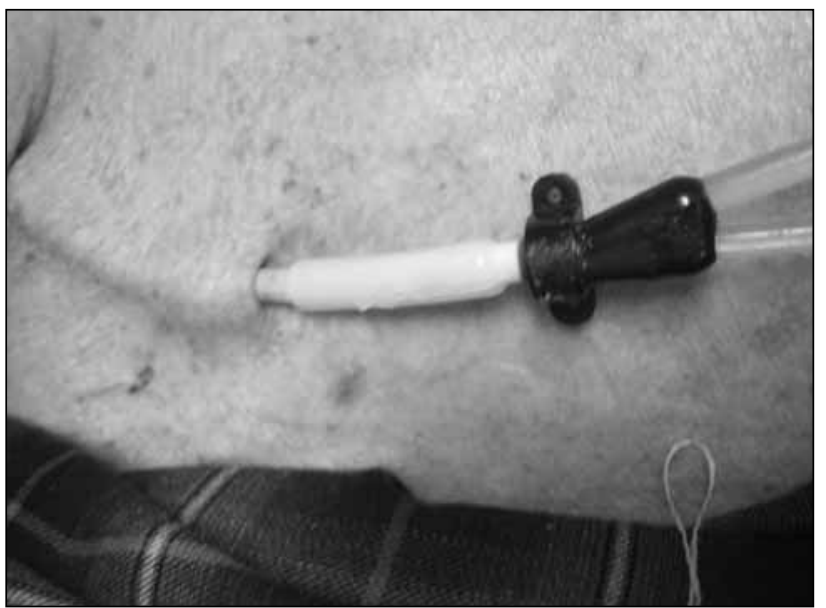

Figura 4.

\section{Resultados}

Datos obtenidos a partir del año 2006: (Figura 5)

\begin{tabular}{|c|c|c|c|c|}
\hline Sex & Age & $\begin{array}{c}\text { Catheter } \\
\text { Reparation } \\
\text { Date }\end{array}$ & $\begin{array}{c}\text { Catheter } \\
\text { Removal Date }\end{array}$ & Complications \\
\hline 40 & $23-09-2009$ & In use & $\varnothing$ \\
\hline 71 & $11-03-2006$ & $\begin{array}{c}16-12-2006 \\
\text { Infection not } \\
\text { related with reparation }\end{array}$ & $\varnothing$ \\
\hline 68 & $21-10-2006$ & $\begin{array}{c}11-3-2010 \\
\text { Exitus }\end{array}$ & Pressure Ulcer \\
\hline
\end{tabular}

Figura 5.

Hombre de 71 años que presentó fuga durante la sesión de HD el 11/03/2006 y cuyo catéter fue retirado tras una infección no relacionada con la reparación el 16/12/2006.

Hombre de años 68 que presentó fuga durante la sesión de HD el 21/10/2006 y cuyo catéter fue retirado por éxitus del paciente el 13/03/2010. Presentó como complicación una pequeña úlcera por decúbito que se solucionó con un apósito de protección.

Mujer de 40 años que presentó fuga durante la sesión de HD el 23/09/2009 y cuyo catéter continúa en uso en la actualidad. No presenta complicaciones.

\section{Discusión}

La eficacia de la técnica parece constatarse por la larga duración de los catéteres tras la reparación: en caso de no haberse realizado, éstos tendrían que haber sido reemplazados de inmediato o en su defecto habría sido necesaria otra vía para seguir realizando HD periódicas.

El tiempo de funcionamiento fue variable, desde 9 meses hasta más de 3 años, estando uno de ellos en uso actualmente.

La retirada del catéter no estuvo relacionada en ningún caso con la técnica de reparación efectuada y sólo se registró una complicación menor en uno de los casos, una úlcera por presión sin mayores consecuencias.

Hoy en día existe gran variedad de elementos y kits de reparación enfocados sobre todo para DP, pero no así para los catéteres de HD. Creemos necesario el desarrollo de la investigación en este campo, al quedar demostrada su utilidad, así como su divulgación entre todos los profesionales de la enfermería nefrológica.

Por ello, proponemos realizar un estudio más amplio que permita valorar las diferentes técnicas de reparación que se utilizan en las unidades de hemodiálisis de toda España para poder extrapolar los resultados presentados en este artículo.

Recibido: 10 septiembre 2013

Revisado: 20 Septiembre 2013

Modificado: 25 Septiembre 2013

Aceptado: 26 Septiembre 2013

\section{Bibliografía}

1. López Gómez, JM. Avances en hemodiálisis clínica: accesos vasculares y pacientes de edad avanzada. Nefrología 2008; Supl.5:67-70.

2. Rodríguez-Hernández, JA et al. Guía de acceso vascular en hemodiálisis. Nefrología, Vol.25 Supl.1.

3. NKF/DOQI: Clinical Practice Guidelines for Vascular Access. Am J. Kidney Dis 2001; 37 Supl.1:S137S181. 